\title{
Optimum dosage of ispaghula husk in patients with irritable bowel syndrome: correlation of symptom relief with whole gut transit time and stool weight
}

\author{
ASHWINI KUMAR, NIRMAL KUMAR, J C VIJ, S K SARIN, AND B S ANAND \\ From the Department of Gastroenterology, GB Pant Hospital, New Delhi, India
}

SUMmaRY To determine the optimum dose of ispaghula husk in patients with irritable bowel syndrome (IBS) and to assess the correlation, if any between the relief in patients' symptoms and the whole gut transit time, and the increase in stool weight, a two part study was carried out. In part 1,14 male patients were given ispaghula husk in increasing doses of $10 \mathrm{~g}, 20 \mathrm{~g}$, and $30 \mathrm{~g}$ a day for a duration of 17 days each (14 days of study period +three days of stool collection). Ten patients completed the trial. The symptom score improved significantly with all the three doses of ispaghula. Both $20 \mathrm{~g}$ and $30 \mathrm{~g}$ doses of ispaghula were superior to the $10 \mathrm{~g}$ dose but there was no significant difference between the $20 \mathrm{~g}$ and $30 \mathrm{~g}$ doses. There was a significant $(\mathrm{p}<0.001)$ increase in the daily stool weight with $10 \mathrm{~g}$ dose of fibre with further significant increases with the $20 \mathrm{~g}$ and $30 \mathrm{~g}$ doses. A positive correlation was seen between the improvement in the symptom score and the increase in stool weight with the $10 \mathrm{~g}$ dose of ispaghula but not with the $20 \mathrm{~g}$ and $30 \mathrm{~g}$ doses. Whole gut transit time remained fairly constant throughout the study period and there was no relationship with either the dose of ispaghula, the alteration in stool weight, or the improvement in the patients symptoms. Ten patients completed part 2 of the study in which ispaghula husk was given in the same dose $(10 \mathrm{~g}, 20 \mathrm{~g}$, and $30 \mathrm{~g})$ but in a random order and with a 'washout' period of one week between individual doses. Again all the three doses of ispaghula produced a significant improvement in the symptoms; $20 \mathrm{~g}$ and $30 \mathrm{~g}$ doses were equally effective and both were significantly superior to the $10 \mathrm{~g}$ dose. Assessed individually, all the three symptoms improved significantly; improvement in constipation and pain abdomen was more pronounced than diarrhoea. It is concluded that the optimum dose of ispaghula husk in irritable bowl syndrome is $20 \mathrm{~g}$ per day. There is some correlation between the increase in stool weight and the improvement in symptom score but the whole gut transit time remains unchanged despite alterations in stool weight and patients' symptoms.

Some studies ${ }^{1-5}$ but not others ${ }^{67}$ show that administration of fibre, results in significant improvement in the symptoms of patients with irritable bowel syndrome. This beneficial effect has been noted not only in the west but also in India ${ }^{24}$ where the diet is comparatively richer in its fibre content. ${ }^{8}$ Furthermore, fibre is useful whether it is used alone or in combination with

Address for correspondence: Dr B S Anand, Department of Gastroenterology, GB Pant Hospital, New Delhi, 110002 , India.

Received for publication 13 June 1986. other drugs. ${ }^{34}$ A number of aspects of the therapeutic role of fibre in irritable bowel syndrome remain to be established, however, for example, the optimum dose of fibre in irritable bowel syndrome has not been determined. Moreover, the effect of different quantities of fibre on the stool weight and the whole gut transit time, and the relationship of these parameters with the clinical response of the patient has not been determined. To examine these issues the present study was carried out in the form of a dose response therapeutic trial. 


\section{Methods}

\section{PATIENTS}

The study was conducted in two parts; part 2 was designed after part 1 had been completed and the results were available.

\section{PART 1}

Fourteen consecutive male patients with irritable bowel syndrome were included in the study. The diagnosis of irritable bowel syndrome was made on clinical grounds and in the presence of normal routine haematological tests, stool examination, sigmoidoscopy, and rectal biopsy. Barium studies and fibreoptic endoscopy were carried out only if there was clinical suspicion of an organic lesion. Details of the bowel complaints of the patients were recorded on a proforma. Diagnosis of constipation or diarrhoea was made according to the patients' assessment and not according to any standard definition.

\section{Treatment schedule}

After the baseline data had been recorded the patients were started on treatment with ispaghula husk (processed flea seed husk). The patients were given a $5 \mathrm{~g}$ measure and were instructed to take $5 \mathrm{~g}$ twice daily ( $10 \mathrm{~g}$ a day) for 17 days (14 days of treatment and three days of stool collection). The dose of fibre was then increased to $20 \mathrm{~g}$ a day for the next 17 days followed by $30 \mathrm{~g}$ a day for the final 17 days. After each two weeks interval the patient was reassessed according to the schedule described below. Any patient failing to report on the appointed day was excluded from the study. Patients were instructed to stop all other medications and were encouraged to consume their normal diet. None of the patients had been on fibre supplements or any other drug for at least one month before entry to the study.

\section{Clinical assessment}

For the purpose of clinical assessment only three symptoms were considered: constipation, diarrhoea, and abdominal pain. For every $25 \%$ subjective improvement in a particular symptom a score of +1 was given with a maximal attainable score of +4 for any one symptom and a maximal attainable score of +12 for the three symptoms. Similarly, for every $25 \%$ subjective worsening, a score -1 was given to a particular symptom. Thus, all patients started with an initial score of 0 . The response of other symptoms to treatment was also recorded but no symptom score was given to these because of difficulty in making a proper assessment. This was particularly true for incomplete evacuation of stools which was incidently the commonest symptom.

\section{Transit time}

Whole gut transit time. was measured by the method of Hinton et al. ${ }^{9}$ Briefly, the patients were given 25 radio-opaque markers before breakfast. They were instructed to record the exact time of each bowel opening and to collect the entire stool in separate polyethylene bags. Each stool collection was examined under an image intensifier and transit time was defined as the time taken to excrete 20 of the 25 markers.

\section{Stool weight}

The mean daily stool weight was calculated from the total stool passed during $72 \mathrm{~h}$, starting immediately after ingestion of the markers. ${ }^{1810}$

Assessment of the clinical response to treatment was made 14 days after starting a particular dose of ispaghula husk. Stool collection for assessment of transit time and stool weight was started from day 15 to day 17 , while the patient was still on the same dose of fibre.

\section{PART 2}

Nineteen consecutive male patients were included in this part of the study. The criteria of selection and the method of clinical assessment were the same as in part 1. Transit time and stool weight studies were not carried out, however. Each patient received all three doses $(10 \mathrm{~g}, 20 \mathrm{~g}$, and $30 \mathrm{~g} /$ day $)$ of ispaghula husk; the order of administration was randomised according to a pre-arranged table. Treatment was continued for two weeks with a 'wash out' period of one week between individual doses. Clinical assessment was made at the end of each two weeks of study period. The person assessing the clinical status was not informed about the dose of fibre received by the patient.

\section{STATISTICAL ANALYSIS}

Paired Student's $t$ test was applied for comparing stool weight and transit time values with various doses of isphagula husk. The $t$ test value was first validated by carrying out the one-way analysis of variance. Changes in the individual symptom scores with different doses of fibre were compared using the Wilcoxon's signed rank sum test. Because of the skewed distribution of the values of stool weight in the original units, log values were used while assessing the increase in stool weight.

\section{Results}

PART 1

Clinical assessment

Ten of the 14 patients completed the study. The mean $( \pm S D)$ age of the 10 patients was $32.6( \pm 20 \cdot 4)$ years 
Fig. 1 Frequency of various symptoms in 10 patients in part 1 and 10 patients in part 2 of the study.

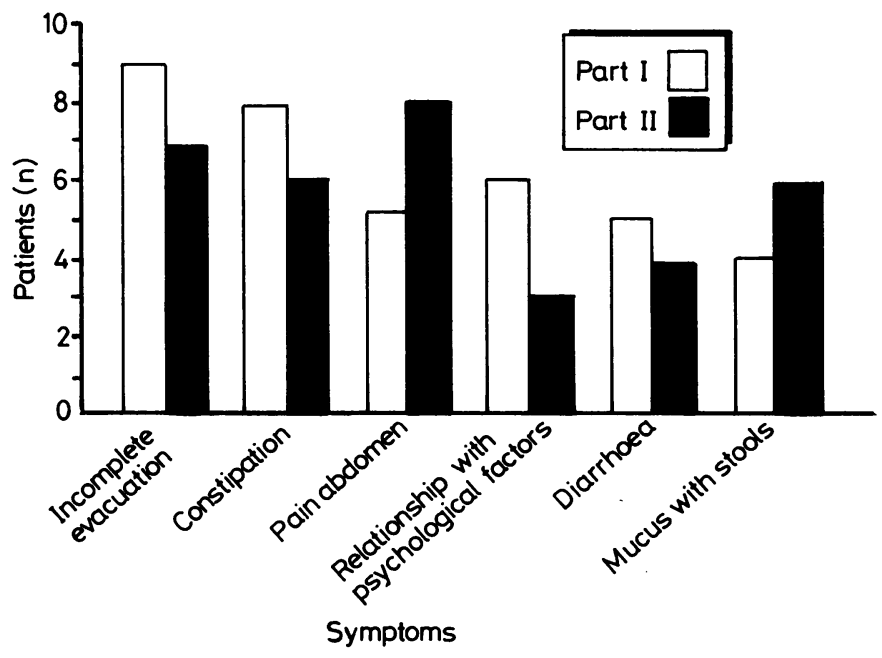

(range 21 to 50 years). The duration of illness before entry into the trial varied from six months to 12 years (mean $\pm \mathrm{SD}=3 \cdot 7 \pm 3.5$ years).

The most common symptom (nine of 10 patients) was incomplete evacuation of the bowel (Fig. 1). This symptom was present irrespective of whether the patient complained of diarrhoea or constipation. The next commonest symptom was constipation (eight) followed by abdominal pain (five), diarrhoea (five), passage of mucus in stools (four) and abdominal distension (three); the overlap of figures is because a number of patients had more than one symptom. Six patients noted a close relationship between the bowel complaints and emotional stress.

After treatment with $10 \mathrm{~g}$ ispaghula husk for two weeks, there was a significant improvement $(p<0.01)$ in the symptom score (mean $\pm S D=4 \cdot 5 \pm 2 \cdot 8$ ) (Fig. 2). When the amount of fibre was increased to $20 \mathrm{~g}$ a day the sympton score showed a further improvement (mean $\pm \mathrm{SD}=8 \cdot 9 \pm 3 \cdot 1$ ) and the difference from the 10 $\mathrm{g}$ dose was highly significant $(\mathrm{p}<0 \cdot 001)$. A further increase in the amount of fibre to $30 \mathrm{~g}$ a day resulted in only a marginal improvement (mean $\pm \mathrm{SD}=$ $9 \cdot 45 \pm 2 \cdot 1$ ) over the $20 \mathrm{~g}$ dose, the difference being not significant. All four patients who dropped out of the study did so while on the $30 \mathrm{~g}$ dose and complained of difficulty in consuming such large quantities of fibre. There were no fibre-related unwanted effects and all patients who completed the trial tolerated it well.

\section{Stool weight}

The pretreatment stool weight showed marked variations in different patients (range $90 \mathrm{~g}-493 \mathrm{~g}$ ). After administration of ispaghula husk there was a significant $(p<0.001)$ increase in the stool weight over the base line value (Table). Again there was a wide variation in the increase in stool weight in different patients (range $30 \mathrm{~g}-325 \mathrm{~g}$ ). With $20 \mathrm{~g}$ ispaghula the
Fig. 2 Improvement in symptom score with different doses of ispaghula husk (mean $\pm S D$ ). Value on top of the bars indicates the difference from the base line.

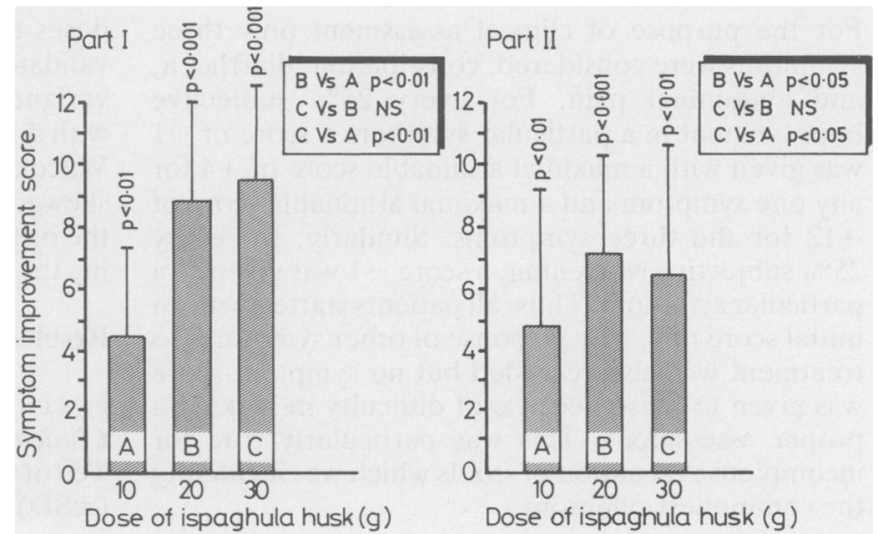


Table Base line stool weight $(\mathrm{g})$ and after two weeks of treatment with varying doses of ispaghula husk in 10 patients with irritable bowel syndrome

\begin{tabular}{|c|c|c|c|c|c|c|c|c|}
\hline \multirow[t]{2}{*}{ Caseno. } & \multirow{2}{*}{$\begin{array}{l}\text { Presenting } \\
\text { complaint }\end{array}$} & \multicolumn{4}{|l|}{ Stool weight } & \multicolumn{3}{|c|}{ Difference in stool weight } \\
\hline & & $B L$ & $\boldsymbol{A}$ & $\boldsymbol{B}$ & $C$ & $A-B L$ & $B-A$ & $C-B$ \\
\hline 1 & Dia/Con & 157 & 292 & 320 & 343 & 135 & 28 & 23 \\
\hline 2 & Dia/Con & 137 & 323 & 340 & 340 & 186 & 17 & 0 \\
\hline 3 & Dia/Con & 285 & 315 & 336 & 410 & 30 & 21 & 74 \\
\hline 4 & Con & 287 & 610 & 670 & 700 & 323 & 60 & 30 \\
\hline 5 & Con & 90 & 415 & 482 & 480 & 325 & 67 & -2 \\
\hline 6 & Con & 263 & 320 & 363 & 430 & 57 & 43 & 67 \\
\hline 7 & Con & 343 & 410 & 428 & 496 & 67 & 18 & 68 \\
\hline 8 & Con & 297 & 338 & 410 & 531 & 41 & 72 & 121 \\
\hline 9 & Dia & 493 & 547 & 654 & 650 & 54 & 107 & -4 \\
\hline 10 & Dia & 483 & 557 & 690 & 710 & 74 & 133 & 20 \\
\hline Mean $\pm S I$ & & $283 \cdot 5 \pm 134 \cdot 5$ & $412 \cdot 7 \pm 117 \cdot 4$ & $469 \cdot 3 \pm 147 \cdot 7$ & $509 \cdot 0 \pm 137 \cdot 6$ & $129 \cdot 2 \pm 112 \cdot 8$ & $56 \cdot 6 \pm 39 \cdot 5$ & 39 \\
\hline
\end{tabular}

Con $=$ Constipation. Dia $=$ Diarrhoea. Dia $/$ Con $=$ Alternating diarrhoea and constipation. $A=10 \mathrm{~g} /$ day of ispaghula husk. $B=20 \mathrm{~g} /$ day $\mathrm{C}=30 \mathrm{~g} / \mathrm{day} . \mathrm{BL}=$ Base line value of stool weight

stool weight showed a further significant increase $(\mathrm{p}<0.01)$ over the values attained with the $10 \mathrm{~g}$ dose (Fig. 3). The magnitude of increase with $20 \mathrm{~g}$, however, was less than that obtained with the $10 \mathrm{~g}$ dose of fibre. With $30 \mathrm{~g}$ ispaghula husk there was a further significant $(p<0.05)$ increase in stool weight over the values obtained with $20 \mathrm{~g}$. The magnitude of increase in stool weight with $30 \mathrm{~g}$ showed a further decline compared with the increase with $20 \mathrm{~g}$ dose.

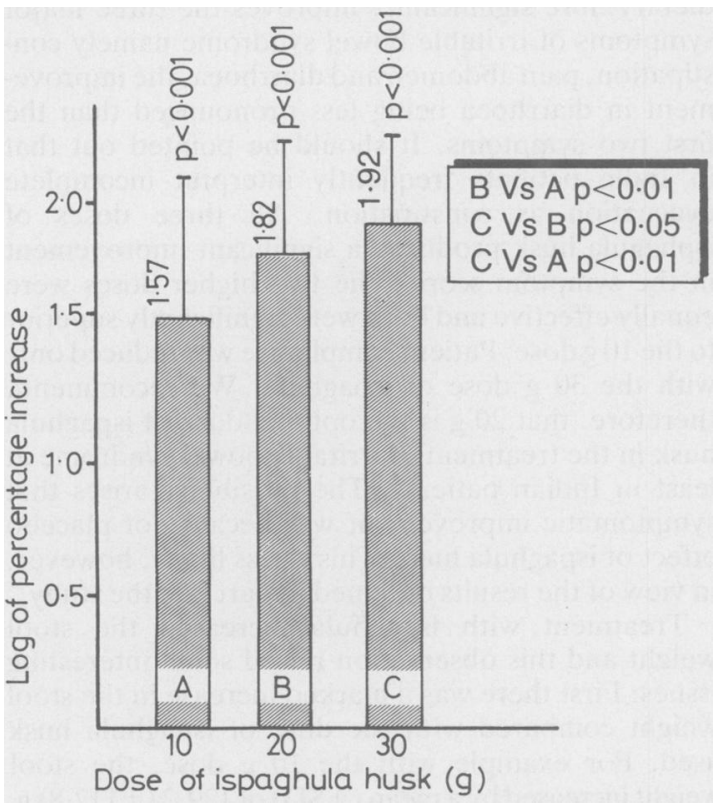

Fig. 3 Mean ( $\pm S D)$ log of percentage increase in stool weight with different doses of ispaghula husk. Value on the top of the bars indicates the difference from the base line values.
A definite correlation was obtained between the increase in stool weight and the improvement in the symptom score with the $10 \mathrm{~g}$ dose of fibre $(\mathrm{r}=0 \cdot 64$, $\mathrm{p}<0.05)$, however, no such correlation was noted with the $20 \mathrm{~g}(\mathrm{r}=0.56)$ and $30 \mathrm{~g}(\mathrm{r}=0.09)$ doses.

\section{Transit time}

The base line mean ( $\pm \mathrm{SD}$ ) whole gut transit time was $(27 \cdot 8 \pm 10.98 \mathrm{~h}$; range: $19-45 \cdot 75 \mathrm{~h})$. After $10 \mathrm{~g}$ ispaghula husk, there was no appreciable change in the transit time $(28.33 \pm 9.7 \mathrm{~h}$; range $21-44.5 \mathrm{~h})$. Similar findings were obtained with $20 \mathrm{~g}(28 \cdot 12 \pm$ 9.37; range $21-43.5 \mathrm{~h})$ and $30 \mathrm{~g} \cdot(28.31 \pm 9.79$; range 20.2-43.0 h) doses of ispaghula husk and the differences from the base line values remained statistically insignificant.

\section{PART 2}

Nine of the 19 patients failed to complete the study, two on $10 \mathrm{~g}$, three on $20 \mathrm{~g}$, and four on $30 \mathrm{~g}$ dose of ispaghula husk. The age of the patients (mean $\pm \mathrm{SD}=$ $26.7 \pm 4.7$ years) and the duration of illness before entry into the trial $(4 \cdot 32 \pm 4 \cdot 2$ years; range $2-12$ years $)$ were comparable with those in part 1 of the study. The commonest symptoms in this group was abdominal pain (eight), followed by incomplete evacuation of bowel (seven), constipation (six), passage of mucus in stools (six), diarrhoea (four), and distention of abdomen (two). Three patients noted an association between the bowel complaints and emotional stress.

\section{Clinical assessment}

The results of symptomatic improvement were similar to those obtained in part 1 of the study (Fig. $2)$. After two weeks of treatment, the mean $( \pm S D)$ total symptom scores were: $4.8( \pm 4.4)$ with $10 \mathrm{~g}$ dose 
of fibre, $7 \cdot 15( \pm 3 \cdot 72)$ with $20 \mathrm{~g}$ and $6 \cdot 4( \pm 4 \cdot 25)$ with $30 \mathrm{~g}$. All three doses produced a statistically significant $(\mathrm{p}<0.01$ for $10 \mathrm{~g}$ and $30 \mathrm{~g} ; \mathrm{p}<0.001$ for $20 \mathrm{~g}$ ) improvement in the symptom score. Again, the $20 \mathrm{~g}$ and $30 \mathrm{~g}$ doses of ispaghula were superior $(\mathrm{p}<0.05$ for each) to the $10 \mathrm{~g}$ dose while there was no significant difference between $20 \mathrm{~g}$ and $30 \mathrm{~g}$ doses. None of the patients in this group complained of any fibre related unwanted effects.

\section{Effect of ispaghula husk on individual symptoms}

The effect of ispaghula on individual symptoms was also assessed. For this purpose, the results of part 1 and 2 were combined so as to obtain a sufficient number of subjects in each group. The results are shown in Fig. 4. All the three doses of fibre produced a significant improvement ( $p<0.001$ for each dose) in constipation. Both $20 \mathrm{~g}$ and $30 \mathrm{~g}$ doses were superior to the $10 \mathrm{~g}$ dose $(\mathrm{p}<0.001$ and $<0.01$ respectively) but there was no difference between the two larger doses. Similar results were obtained in the relief of abdominal pain. Improvement in diarrhoea was also statistically significant with each dose of ispaghula but the response was less pronounced compared with the other two symptoms; $20 \mathrm{~g}$ was superior to the $10 \mathrm{~g}$ dose of fibre but there was no difference between $10 \mathrm{~g}$ and $30 \mathrm{~g}$ doses.

\section{Discussion}

In part 1 of the study ispaghula husk was administered successfully in gradually increasing quantities of $10 \mathrm{~g}, 20 \mathrm{~g}$, and $30 \mathrm{~g}$ with no 'wash out' period between the doses. Such a protocol had the theoretical disadvantage that the effect of one dose of fibre could 'spill over' to the next dose and thus influence the final result. To obviate this, in part 2 , the three doses of ispaghula were given in a random order and in a blind fashion with a 'wash out' period of one week between two doses. Only male patients were included in the study because it has been observed that women show alterations in the bowel habit during the course of a menstrual cycle. ${ }^{11}$

The beneficial effect of placebo in irritable bowel syndrome has been well described. ${ }^{67}$ We did not include a placebo control group, however, for various reasons: (a) it is difficult to devise an 'inert' material which can be made to resemble ispaghula husk, (b) fibre can be given in capsules but it would require approximately 90 capsules a day to administer a dose of $30 \mathrm{~g}$, (c) another method of disguising fibre is to administer it as biscuits but this requires cooking and defeats the purpose of testing a material in a ('natural') form in which it is normally going to be consumed and (d) moreover, the main purpose of the present study was to determine the optimum dose

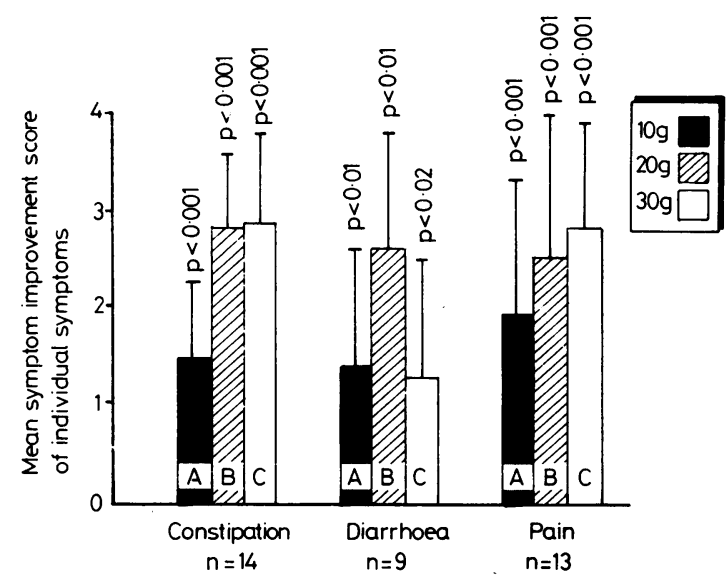

Fig. 4 Improvement in symptom score of individual symptoms with different doses of ispaghula husk (mean $\pm S D)$. Value on top of the bars indicates the difference from the base line values. For constipation, $A$ vs $B p<0.001$, $A$ vs $C p<0.01$ and $B$ vs $C N S$ : for pain, $A$ vs $B p<0.05$, $A$ vs $C p<0.05$ and $B$ vs $C N S$; for diarrhoea $A$ vs $B p<0.02$, $A$ vs $C N S$ and $B$ vs $C N S$.

ispaghula husk for which each subject acted as his own control and thus a placebo group was superfluous.

The results of both parts of the study indicate that dietary fibre significantly improves the three major symptoms of irritable bowel syndrome namely constipation, pain abdomen and diarrhoea, the improvement in diarrhoea being less pronounced than the first two symptoms. It should be pointed out that in India patients frequently interpret incomplete evacuation as constipation. All three doses of isphagula husk produced a significant improvement in the symptom score. The two higher doses were equally effective and both were significantly superior to the $10 \mathrm{~g}$ dose. Patient compliance was reduced only with the $30 \mathrm{~g}$ dose of ispaghula. We recommend, therefore, that $20 \mathrm{~g}$ is the optimal dose of ispaghula husk in the treatment of irritable bowel syndrome at least in Indian patients. The possibility arises that symptomatic improvement was because of placebo effect of ispaghula husk. This is less likely, however, in view of the results obtained in part 2 of the study.

Treatment with ispaghula increased the stool weight and this observation raised some interesting issues. First there was a marked increase in the stool weight compared with the dose of ispaghula husk used. For example with the $10 \mathrm{~g}$ dose, the stool weight increased by a mean $( \pm S D)$ of $129 \cdot 2( \pm 112 \cdot 8) \mathrm{g}$. Similar observations were made previously by Williams and Olmsted ${ }^{12}$ in their metabolic balance experiments. They noted that each gram of fibre 
increased the stool weight by as much as $12 \mathrm{~g}$ to $20 \mathrm{~g}$. The exact mechanism by which dietary fibre increases the stool weight is not entirely clear as this increase is much more than can be explained by simple water absorption alone. ${ }^{12}$ The second interesting observation was that the increase in stool weight had no influence on the transit time. Thus, contrary to popular belief, dietary fibre appeared not to have any effect on the time taken by food to traverse the gastrointestinal tract which remained fairly constant in the same individual. Further more, the transit time was not related to the bowel habit of the patient (diarrhoea or constipation) or to the response to treatment (improved, no effect, or worsened). The third observation of interest was the association between the increase in stool weight and the improvement in the symptom score. A positive correlation was seen with the $10 \mathrm{~g}$ dose of fibre $(r=0.64)$. With the $20 \mathrm{~g}$ dose the correlation coefficient just failed to reach levels of statistical significance $(r=0.56)$ while no correlation was observed with $30 \mathrm{~g}$ of fibre $(\mathrm{r}=0.09)$. Thus there is some association, although not a strong one, between the increase in the bulk of intestinal contents and relief of symptoms in irritable bowel syndrome.

We had a high drop out rate of $39 \%$ (13 out of 33 ). Most of these patients $(8 ; 61.5 \%)$ dropped out while on the $30 \mathrm{~g}$ dose because they found it difficult to consume such large quantities of fibre. The remaining five (two on $10 \mathrm{~g}$ and three on $20 \mathrm{~g}$ ) dropped out because they felt sufficiently well and did not wish to carry on with the study.

It is concluded that the optimum dose of ispaghula husk in the treatment of irritable bowel syndrome is $20 \mathrm{~g}$ per day. The mechanism by which dietary fibre improves the patients symptoms is related to some extent to the increase in the stool weight but is independent of the whole gut transit time of food.

\section{References}

1 Cann PA, Read NW, Holdworth CD. What is the benefit of Coarse wheat bran in patients with irritable bowel syndrome? Gut 1984; 25: 168-73.

2 Golecha AC, Chadda VS, Chadda S, Sharma SK, Mishra SN. Role of ispaghula husk in the management of irritable bowel syndrome: A randomised double blind cross over study. J Assoc Physicians India 1982; 30: 353-5.

3 Ritchie JA, Truelove SC. Treatment of irritable bowel syndrome with lorazepam, hyoscine butyl bromide and ispaghula husk. $\mathrm{Br}$ Med J 1979; 1: 376-8.

4 Nigam P, Kapoor KK, Rastog CK, Kumar A, Gupta AK. Different therapeutic regimens in irritable bowel syndrome. J Assoc Physicians India 1984; 32: 1041-44.

5 Manning AP, Heaton KW, Harvey RF, Uglow P. Wheat fibre and the irritable bowel syndrome. Lancet 1977; 2: 417-8.

6 Soltoff JI, Gudmand Hayer E, Kreg B, Kristensen E, Wolff HR. A double blind trial of wheat bran on symptoms of irritable bowel syndrome. Lancet 1976; 1: 270-2.

7 Longstreth GF, Fox DD, Youkeles L, Fortsythe AS, Wolochow DA. Psyllium therapy in irritable bowel syndrome. A double blind trial. Ann Intern Med 1981; 95: 53-6.

8 Tandon RK, Prasad Nisha, Gupta MC, Tandon BN. Stool weight and transit time in North Indians. $J$ Assoc Physicians India 1976; 24: 807-10.

9 Hinton JM, Lennard-Jones JE, Young AC. A new method for studying gut transit time using radio-opaque markers. Gut 1969; 10: 842-7.

10 Hillman LC, Stace NH, Pomare EW. Irritable bowel patients and their long term response to a high fibre diet. Am J Gastroenterol 1984; 79: 1-6.

11 Rees WDW, Rhodes J. Altered bowel habit and menstruation. Lancet 1976; 289: 475.

12 Williams RD, Olmsted WH. The effect of cellulose, hemicellulose and lignin on the weight of the stool. A contribution to the study of laxation in man. $J$ Nutr 1936; 11: 433-49. 